\title{
ON THE CONNECTIVITY OF BRANCH LOCI OF MODULI SPACES
}

\author{
Gabriel Bartolini, Antonio F. Costa* and Milagros Izquierdo ${ }^{\dagger}$ \\ Linköpings Universitet, Matematiska Institutionen \\ 58183 Linköping, Sweden; gabriel.bartolini@liu.se \\ UNED, Facultad de Ciencias, Departamento de Matemáticas Fundamentales \\ 28040 Madrid, Spain; acosta@mat.uned.es \\ Linköpings Universitet, Matematiska Institutionen \\ 58183 Linköping, Sweden; milagros.izquierdo@liu.se
}

\begin{abstract}
The moduli space $\mathcal{M}_{g}$ of compact Riemann surfaces of genus $g$ has orbifold structure and the set of singular points of the orbifold is the branch locus $\mathcal{B}_{g}$. In this article we show that $\mathcal{B}_{g}$ is connected for genera three, four, thirteen, seventeen, nineteen and fiftynine, and disconnected for any other genus. In order to prove this we use Fuchsian groups, automorphisms of order 5 and 7 of Riemann surfaces, and calculations with GAP for some small genera.
\end{abstract}

\section{Introduction}

The moduli space $\mathcal{M}_{g}$ of compact Riemann surfaces of genus $g$ is the (discontinuous) quotient of the Teichmüller space by the action of mapping class group. Hence it has the structure of a complex orbifold, whose set of singular points is the branch locus $\mathcal{B}_{g}$. The set $\mathcal{B}_{g}$ consists of the surfaces with non-trivial automorphisms, this fact makes the study of $\mathcal{B}_{g}$ interesting. In this article we study the connectedness of $\mathcal{B}_{g}$.

Our main tool will be the uniformization of Riemann surfaces by Fuchsian groups. Given a Riemann surface $X$ of genus $g>1$, we consider the universal covering $\mathcal{H} \rightarrow X$, where $\mathcal{H}$ is the upper half plane and the cover transformations of this map is isomorphic to $\pi_{1}(X)$. Hence there is a representation $r: \pi_{1}(X) \rightarrow \operatorname{Isom}^{+}(\mathcal{H})=$ $P S L(2, \mathbf{R})$ such that $X=\mathcal{H} / r\left(\pi_{1}(X)\right)$ and $r\left(\pi_{1}(X)\right)$ is a discrete subgroup of $P S L(2, \mathbf{R})$ (i.e. a Fuchsian group).

If there is $\gamma \in P S L(2, \mathbf{R})$, such that $r_{1}\left(\pi_{1}(X)\right)=\gamma\left(r_{2}\left(\pi_{1}(X)\right)\right) \gamma^{-1}$, clearly the Fuchsian groups $r_{1}\left(\pi_{1}(X)\right)$ and $r_{2}\left(\pi_{1}(X)\right)$ uniformize the same marked Riemann surface and conversely, conformally equivalent marked surfaces have conjugate Fuchsian uniformization groups. The space

$\left\{r: \pi_{1}(X) \rightarrow P S L(2, \mathbf{R}): \mathcal{H} / r\left(\pi_{1}(X)\right)\right.$ is a genus $g$ surface $\} /$ conjugation in $P S L(2, \mathbf{R})$ is the Teichmüller space $\mathbf{T}_{g}$. The Teichmüller space $\mathbf{T}_{g}$ has complex structure of dimension $3 g-3$ and it is simply connected.

doi:10.5186/aasfm.2013.3820

2010 Mathematics Subject Classification: Primary 14h15, 30F10, 32 G15.

Key words: Branch locus, moduli space, equisymmetric stratification, automorphisms of Riemann surfaces.

*Partially supported by MTM2011-23092.

$\dagger$ Partially supported by the Swedish Research Council (VR), support by the Institut MittagLeffler (Djursholm, Sweden) is gratefully acknowledged. 
The group Aut $^{+}\left(\pi_{1}(X)\right) / \operatorname{Inn}\left(\pi_{1}(X)\right)=\operatorname{Mod}_{g}$ is the modular group or mapping class group, acting by composition on $\mathbf{T}_{g}$. Now we define the moduli space by $\mathcal{M}_{g}=$ $\mathbf{T}_{g} / \operatorname{Mod}_{g}$ and $\mathcal{M}_{g}$ is the space of complex structures on surfaces of genus $g$.

The projection $\mathbf{T}_{g} \rightarrow \mathcal{M}_{g}=\mathbf{T}_{g} / \operatorname{Mod}_{g}$ is a regular branched covering with branch locus $\mathcal{B}_{g}$, in other words, $\mathcal{M}_{g}$ is an orbifold with singular locus $\mathcal{B}_{g}$. The branch locus $\mathcal{B}_{g}$ consists of the Riemann surfaces with symmetry, i.e. Riemann surfaces with nontrivial automorphism group (except when $g=2$, where $\mathcal{B}_{2}$ consists of the surfaces with automorphisms different from the hyperelliptic involution and the identity). Our goal is the study of the topology of $\mathcal{B}_{g}$. The study of $\mathcal{B}_{g}$ started at the 60's and 70's see, for instance: $[\mathrm{R}]$ and $[\mathrm{H}]$.

As a prototype, let us describe $\mathcal{B}_{1}$. Each elliptic Riemann surface is uniformized by a lattice $\left\{n z_{1}, m z_{2}: n, m \in \mathbf{Z}\right.$, with $\left.\operatorname{Im}\left(z_{1} / z_{2}\right)>0\right\}$, that can be normalized and parametrized by a complex number, the modulus of the basis $\left\{z_{1}, z_{2}\right\}: z_{1} / z_{2}=\tau \in$ $\{z \in \mathbf{C}: \operatorname{Im} z>0\}$. Two lattices uniformize equivalent elliptic Riemann surfaces if there are related by the action of $\operatorname{PSL}(2, \mathbf{Z})$. Hence the moduli space in this case is the orbifold

$$
\mathcal{M}_{1}=\{z \in \mathbf{C}: \operatorname{Im} z>0\} / P S L(2, \mathbf{Z})
$$

that is the Riemann sphere with a cusp and two cone points: $[i]$, with isotropy group of order 2 , and $\left[e^{2 \pi i / 3}\right]$ with isotropy group of order 3 . Then $\mathcal{B}_{1}=\left\{[i],\left[e^{2 \pi i / 3}\right]\right\}$ is disconnected.

The connectedness of moduli spaces of hyperelliptic, $p$-gonal and real Riemann surfaces has been widely studied, for instance by [BSS], [CI1], [K], [G], [Se], [BCIP], [BCI], [BEMS].

It is known that $\mathcal{B}_{2}$ is not connected, since Bolza and later Kulkarni (see [Bo], $[\mathrm{K}]$ and $[\mathrm{BuCI}]$ ) showed that the curve $w^{2}=z^{5}-1$ is isolated in $\mathcal{B}_{2}$, i.e. this single surface is a connected component of $\mathcal{B}_{2}$, furthermore $\mathcal{B}_{2}$ has exactly two connected components (see $[\mathrm{BI}]$ ). The branch loci $\mathcal{B}_{3}, \mathcal{B}_{4}$ and $\mathcal{B}_{7}$ are connected (see [BCIP] and $[\mathrm{CI} 2]$ ) and $\mathcal{B}_{5}, \mathcal{B}_{6}, \mathcal{B}_{8}$ are connected with the exception of isolated points (see [BI]). In this paper we established that the connectedness of the branch loci is a phenomenon for low genera. More precisely we prove that $\mathcal{B}_{g}$ is disconnected for $g \geq 60$ and, in the last section, we prove, using GAP ([GAP]), that the only genera $g$ where $\mathcal{B}_{g}$ is connected are exactly $g=3,4,13,17,19,59$.

\section{Symmetric Riemann surfaces}

Let $X$ be a Riemann surface, with genus $g>2$, and assume that $\operatorname{Aut}(X) \neq\{1\}$. Hence $X / \operatorname{Aut}(X)$ is an orbifold and there is a Fuchsian group $\Gamma \leq P S L(2, \mathbf{R})$, such that

$$
\mathcal{H} \rightarrow X=\mathcal{H} / \pi_{1}(X) \rightarrow X / \operatorname{Aut}(X)=\mathcal{H} / \Gamma
$$

The algebraic structure of $\Gamma$ is given by the signature $s(\Gamma)=\left(h ; m_{1}, \ldots, m_{r}\right)$, where $h$ is the genus of $\mathcal{H} / \Gamma$ and $m_{1}, \ldots, m_{r}$ are the orders of the cone points of the orbifold $\mathcal{H} / \Gamma$. The signature determines the algebraic structure of $\Gamma$ and a canonical presentation:

$$
\left\langle a_{i}, b_{i}, i=1, \ldots, h, x_{i}, i=1, \ldots, r: \prod_{i=1}^{h}\left[a_{i}, b_{i}\right] \prod_{i=1}^{r} x_{i}=1, x_{i}^{m_{i}}=1\right\rangle
$$

where the $a_{i}, b_{i}$ are hyperbolic transformations and the $x_{i}$ are elliptic transformations. 
Each type of Fuchsian groups with fixed signature determines a Teichmüller space. Let $\mathcal{G}$ be an abstract group with presentation $\left(^{*}\right)$ and then isomorphic to all the Fuchsian groups of signature $s=\left(h ; m_{1}, \ldots, m_{r}\right)$. Hence the Teichmüller space of Fuchsian groups of signature $s$ is $\{r: \mathcal{G} \rightarrow P S L(2, \mathbf{R}): s(r(\mathcal{G}))=s\} /$ conjugation in $P S L(2, \mathbf{R})=\mathbf{T}_{s}$ (see $\left.[\mathrm{MS}]\right)$.

The Teichmüller space $\mathbf{T}_{s}$ is a contractible complex manifold of dimension $3 g-$ $3+r$. An action of a group $G$ on a surface $X$ is a monomorphism

$$
\alpha: G \rightarrow\{\text { (orientation preserving) homeomorphisms } X \rightarrow X\} .
$$

Given two actions $\alpha$ and $\alpha^{\prime}$ of two groups $G$ and $G^{\prime}$ on two surfaces $X$ and $X^{\prime}$, we say the actions are topologically equivalent if there is an (orientation preserving) homeomorphism $h: X \rightarrow X^{\prime}$ such that $h^{-1} \alpha^{\prime}\left(G^{\prime}\right) h=\alpha(G)$.

If genus $(X)=g$ and $\pi_{1}(X) \subset \mathcal{G}$ there is a natural monomorphism $i: \mathbf{T}_{s} \subset$ $\mathbf{T}_{(g ;-)}=\mathbf{T}_{g}$, given by the restriction, more precisely to $r: \mathcal{G} \rightarrow \operatorname{PSL}(2, \mathbf{R}), \pi_{1}(X) \subset$ $\mathcal{G}, r(\mathcal{G})$ with signature $s$ corresponds $r^{\prime}=\left.r\right|_{\pi_{1}(X)}: \pi_{1}(X) \rightarrow \operatorname{PSL}(2, \mathbf{R})$, where $r^{\prime}\left(\pi_{1}(X)\right)$ has signature $(g ;-)$ (i.e. is a surface Fuchsian group) (see [H] and [MS]).

If we have $\pi_{1}(X) \triangleleft \mathcal{G}$, then there is an action of the finite group $G=\mathcal{G} / \pi_{1}(X)$ on $X$, given by the representation $\alpha$ of $G$ as the group of cover transformations of $X \rightarrow \mathcal{H} / \Gamma$ where $s(\Gamma)=s(\mathcal{G})$; the above action is determined by the monodromy $a: \mathcal{G} \rightarrow G$. We shall denote $i_{a}$ the inclusion $i_{a}: \mathbf{T}_{s} \rightarrow \mathbf{T}_{g}$ given by the action $\alpha$ determined by $a$. The image of $i_{a}\left(\mathbf{T}_{s}\right)$ by the covering $\mathbf{T}_{g} \rightarrow \mathcal{M}_{g}$ is $\overline{\mathcal{M}}^{G, a}$, where $\overline{\mathcal{M}}^{G, a}$ is the set of Riemann surfaces with automorphisms group containing a subgroup acting in a topologically equivalent way to the action $\alpha$ of $G$ on $X$. The set $\mathcal{M}^{G, a}$ consists of the surfaces $X \in \overline{\mathcal{M}}^{G, a}$ where $\operatorname{Aut}(X)$ acts in a topologically equivalent way to the action $\alpha$ of $G$; thus $\mathcal{M}^{G, a} \subset \overline{\mathcal{M}}^{G, a}$. Notice that $\overline{\mathcal{M}}^{G, a}$ is the topological closure of $\mathcal{M}^{G, a}$ in $\mathcal{M}_{g}$.

Furthermore $\mathcal{M}_{g}=\bigcup \mathcal{M}^{G, a}$ and $\mathcal{B}_{g}=\bigcup_{G \neq\{1\}} \mathcal{M}^{G, a}$, such covers are called the equisymmetric stratifications ([B]), there are also the coverings $\mathcal{M}_{g}=\bigcup \overline{\mathcal{M}}^{G, a}$ and $\mathcal{B}_{g}=\bigcup_{G \neq\{1\}} \overline{\mathcal{M}}^{G, a}$. A set $\overline{\mathcal{M}}^{G, a}$ in the above covering will be called an isolated equisymmetric stratum if it satisfies $\overline{\mathcal{M}}^{G, a} \cap \overline{\mathcal{M}}^{H, b}=\varnothing$, for any other non-trivial group $H$ with an action $b$ on surfaces of genus $g$. Note that for an isolated stratum $\overline{\mathcal{M}}^{G, a}=\mathcal{M}^{G, a}$ and is a connected component of $\mathcal{B}_{g}$ (see $[\mathrm{B}]$ and $[\mathrm{H}]$ ).

Since each non-trivial group $G$ contains subgroups of prime order, we have the following remark:

Remark 2.1. (see $[\mathrm{C}]$ )

$$
\mathcal{B}_{g}=\bigcup_{p \text { prime }} \overline{\mathcal{M}}^{C_{p}, a}
$$

where $\overline{\mathcal{M}}^{C_{p}, a}$ is the set of Riemann surfaces of genus $g$ with an automorphism group containing $C_{p}$, the cyclic group of order $p$, acting on surfaces of genus $g$ in a fixed way given by $a$.

To show that $\mathcal{B}_{g}$ is disconnected we shall find isolated strata. In order to find these strata we give actions (monodromies) of cyclic groups of order five and seven that are not restrictions of actions of larger groups. 
Remark 2.2. The components in $[\mathrm{C}]$ are not connected components, note that in $[\mathrm{C}]$ the author studies the inclusions between the strata $\mathcal{M}^{G, a}$ and not the intersections between the $\overline{\mathcal{M}}^{G, a}$.

\section{The disconnectedness of $\mathcal{B}_{g}$ for $g \geq 60$}

We will see that the connectedness of $\mathcal{B}_{g}$ only happens for low genera. A very important ingredient in the proof of our main theorem is the Castelnuovo-Severi inequality (see 3.5 of [A], p. 21):

Let $X_{g}, X_{h}$ and $X_{k}$ be compact Riemann surfaces of genus $g, h$ and $k$, respectively, such that $X_{g}$ is a covering of $X_{h}$ of prime degree $p$, and a covering of $X_{k}$ of prime degree $q$, then $g \leq p h+q k+(p-1)(q-1)$. As a consequence, if $g>2 p h+(p-1)^{2}$, there is at most one covering $X_{g} \rightarrow X_{h}$ of degree $p$.

Theorem 3.1. $\mathcal{B}_{g}$ is disconnected for $g>64$. Furthermore $\mathcal{B}_{g}$ contains an isolated equisymmetric stratum of complex dimension:

1. $\frac{g}{3}-1$ if $g \equiv 0 \bmod 3$, for $g>36$,

2. $\frac{g-1}{3}$ if $g \equiv 1 \bmod 3$, for $g>50$,

3. $\frac{g+1}{3}$ if $g \equiv 2 \bmod 3$, for $g>64$.

Proof. In order to prove the theorem we shall find an action of $C_{7}$ on surfaces of genus $g$. Such an action is given by automorphisms of order 7 of a surface $X$ in such a way that it is impossible that $\operatorname{Aut}(X) \supsetneqq C_{7}$, i.e. we find an isolated stratum for $\mathcal{B}_{g}$ that consists of Riemann surfaces with group of automorphisms $C_{7}$ (with a fixed action). We construct monodromies $\theta: \Delta(h ; 7, . r ., 7) \rightarrow C_{5}$, where $r=\frac{g-7 h+6}{3}$, $h=0,1,2, \theta\left(x_{i}\right)=\alpha^{t_{i}}, i=1, \ldots, r$. Let $n_{j}=\left|\left\{t_{i}=j ; i=1, \ldots, r\right\}\right|$, then we will define the epimorphism $\theta$ by sending $n_{j}$ of the canonical elliptic generators of $\Delta$ to $\alpha^{j}$.

Notice that the class of an action is independent of the order in which the different powers of $\alpha$ appear as images of elliptic generators and of the images of the hyperbolic generators of $\Delta$ (see $[\mathrm{N}]$ and $[\mathrm{H}]$ ).

Case 1. $g \equiv 0 \bmod 3, g>36$. Let $\Delta$ be a Fuchsian group with signature $(0 ; 7, . r ., 7)$, with $r=\frac{g}{3}+2$.

The monodromies will be given by the generating vector $\left(n_{1} \alpha, n_{2} \alpha^{2}, n_{3} \alpha^{3}, n_{4} \alpha^{4}\right.$, $\left.n_{5} \alpha^{5}, n_{6} \alpha^{6}\right)$. The values of $n_{j}$ for the different monodromies are given according to the congruence of $g$ modulus 7 .

\begin{tabular}{c|c|cccccc}
$g \bmod 7$ & $r \bmod 7$ & $n_{1}$ & $n_{2}$ & $n_{3}$ & $n_{4}$ & $n_{5}$ & $n_{6}$ \\
\hline$g \equiv 0 \bmod 7$ & $r \equiv 2 \bmod 7$ & $(r-9)$ & 3 & 0 & 1 & 5 & 0 \\
\hline$g \equiv 1 \bmod 7$ & $r \equiv 0 \bmod 7$ & $(r-9)$ & 5 & 3 & 1 & 0 & 0 \\
\hline$g \equiv 2 \bmod 7$ & $r \equiv 5 \bmod 7$ & $(r-9)$ & 3 & 0 & 5 & 0 & 1 \\
\hline$g \equiv 3 \bmod 7$ & $r \equiv 3 \bmod 7$ & $(r-9)$ & 0 & 3 & 5 & 1 & 0 \\
\hline$g \equiv 4 \bmod 7$ & $r \equiv 1 \bmod 7$ & $(r-9)$ & 1 & 5 & 3 & 0 & 0 \\
\hline$g \equiv 5 \bmod 7$ & $r \equiv 6 \bmod 7$ & $(r-9)$ & 5 & 3 & 0 & 1 & 0 \\
\hline$g \equiv 6 \bmod 5$ & $r \equiv 4 \bmod 7$ & $(r-9)$ & 1 & 0 & 0 & 3 & 5
\end{tabular}


The Riemann-Hurwitz formula shows that $g$ is the genus of the Riemann surface $X=\mathcal{H} / \operatorname{ker} \theta$, since

$$
7=\frac{2 \operatorname{genus}(X)-2}{-2+\left(\frac{g}{3}+2\right) \frac{6}{7}}
$$

By construction $X$ has an order 7 automorphism $\xi$ with $r$ fixed points. Now we shall show that, under this condition, $X$ has no automorphisms besides $C_{7}=\langle\xi\rangle$.

Since $X \rightarrow X /\langle\xi\rangle$ is a meromorphic function, by the Castelnuovo-Severi inequality and $g>36, X \rightarrow X /\langle\xi\rangle$ is the unique degree seven meromorphic function, therefore $\langle\xi\rangle$ is a normal subgroup of $\operatorname{Aut}(X)$.

If $\operatorname{Aut}(X) \supsetneqq C_{7}$, since $\langle\xi\rangle \triangleleft \operatorname{Aut}(X)$, there is a non-trivial prime order cyclic action $b$ on the orbifold $X /\langle\xi\rangle$, inducing an automorphism $b_{*}: \Delta \rightarrow \Delta$ such that $\theta \circ b_{*}=\eta \circ \theta$ where $\eta$ is an automorphism of $C_{7}$. The orbifold $X /\langle\xi\rangle$ is conformally equivalent to the Riemann sphere; then the automorphism $b$ has two fixed points and all the generic orbits have order $(b)$ elements. By the condition $\theta \circ b_{*}=\eta \circ \theta$ and the definition of $\theta$ above we get that $b$ has more than two fixed points, contradicting the fact that $b$ is not the identity.

Since $\operatorname{dim} \mathbf{T}_{(0 ; 7, \cdots, 7)}=3 \times 0-3+r$ and $r=\frac{g}{3}+2$, the surfaces admitting a cyclic action as above form an isolated stratum $i_{\theta}\left(\mathbf{T}_{(0 ; 7, r ., 7)}\right)$ in the equisymmetric stratification of dimension $\frac{g}{3}-1$.

Case 2. $g \equiv 1 \bmod 3$. Let $\Delta$ be a Fuchsian group with signature $(1 ; 7, . r ., 7)$, with $r=\frac{g-1}{3}$.

The monodromies will be given by the generating vector $\left(1,1, n_{1} \alpha, n_{2} \alpha^{2}, n_{3} \alpha^{3}\right.$, $\left.n_{4} \alpha^{4}, n_{5} \alpha^{5}, n_{6} \alpha^{6}\right)$, where the 1's in the generating vector mean that $\theta\left(a_{1}\right)=\theta\left(b_{1}\right)=1$. The values of $n_{j}$ for the different monodromies are given according to the congruence of $g$ modulus 7 .

\begin{tabular}{c|c|cccccc}
$g \bmod 7$ & $r \bmod 7$ & $n_{1}$ & $n_{2}$ & $n_{3}$ & $n_{4}$ & $n_{5}$ & $n_{6}$ \\
\hline$g \equiv 0 \bmod 7$ & $r \equiv 2 \bmod 7$ & $(r-10)$ & 3 & 1 & 1 & 0 & 5 \\
\hline$g \equiv 1 \bmod 7$ & $r \equiv 0 \bmod 7$ & $(r-10)$ & 3 & 5 & 1 & 0 & 1 \\
\hline$g \equiv 2 \bmod 7$ & $r \equiv 5 \bmod 7$ & $(r-10)$ & 3 & 1 & 0 & 5 & 1 \\
\hline$g \equiv 3 \bmod 7$ & $r \equiv 3 \bmod 7$ & $(r-10)$ & 3 & 1 & 5 & 0 & 1 \\
\hline$g \equiv 4 \bmod 7$ & $r \equiv 1 \bmod 7$ & $(r-10)$ & 3 & 5 & 1 & 1 & 0 \\
\hline$g \equiv 5 \bmod 7$ & $r \equiv 6 \bmod 7$ & $(r-10)$ & 3 & 5 & 0 & 1 & 1 \\
\hline$g \equiv 6 \bmod 5$ & $r \equiv 4 \bmod 7$ & $(r-10)$ & 3 & 1 & 5 & 1 & 0
\end{tabular}

If $X=\mathcal{H} / \operatorname{ker} \theta$, the Riemann-Hurwitz formula says

$$
7=\frac{2 \operatorname{genus}(X)-2}{\left(\frac{g-1}{3}\right) \frac{6}{7}},
$$

so $g=\operatorname{genus}(X)$. By construction $X$ has an order 7 automorphism $\xi$ with $r$ fixed points. By a similar argument to the one used in the above case, by the CastelnuovoSeveri inequality, the definition of $\theta$ and the fact that an automorphism of the torus different from the identity has at most four fixed points, we have that $X$ has no automorphisms besides $C_{7}=\langle\xi\rangle$.

Now $r=\frac{g-1}{3}$ and $\operatorname{dim} \mathbf{T}_{(1 ; 7, r, 7)}=3 \times 1-3+r$, then the surfaces admitting a cyclic action as above form an isolated stratum of complex dimension $\frac{g-1}{3}$. 
Case $3 . g \equiv 2 \bmod 3$. Let $\Delta$ be a Fuchsian group with signature $(2 ; 7, . r ., 7)$, with $r=\frac{g-8}{3}$.

The monodromies will be given by the generating vector $\left(1,1,1,1, n_{1} \alpha, n_{2} \alpha^{2}, n_{3} \alpha^{3}\right.$, $\left.n_{4} \alpha^{4}, n_{5} \alpha^{5}, n_{6} \alpha^{6}\right)$ where the 1 's in the generating vector mean that $\theta\left(a_{1}\right)=\cdots=$ $\theta\left(b_{2}\right)=1$. The values of $n_{j}$ for the different monodromies are given according to the congruence of $g$ modulus 7 .

\begin{tabular}{c|c|cccccc}
$g \bmod 7$ & $r \bmod 7$ & $n_{1}$ & $n_{2}$ & $n_{3}$ & $n_{4}$ & $n_{5}$ & $n_{6}$ \\
\hline$g \equiv 0 \bmod 7$ & $r \equiv 2 \bmod 7$ & $(r-11)$ & 3 & 1 & 1 & 5 & 1 \\
\hline$g \equiv 1 \bmod 7$ & $r \equiv 0 \bmod 7$ & $(r-11)$ & 1 & 3 & 1 & 5 & 1 \\
\hline$g \equiv 2 \bmod 7$ & $r \equiv 5 \bmod 7$ & $(r-11)$ & 5 & 3 & 1 & 1 & 1 \\
\hline$g \equiv 3 \bmod 7$ & $r \equiv 3 \bmod 7$ & $(r-11)$ & 3 & 5 & 1 & 1 & 1 \\
\hline$g \equiv 4 \bmod 7$ & $r \equiv 1 \bmod 7$ & $(r-11)$ & 1 & 1 & 3 & 1 & 5 \\
\hline$g \equiv 5 \bmod 7$ & $r \equiv 6 \bmod 7$ & $(r-11)$ & 1 & 1 & 1 & 3 & 5 \\
\hline$g \equiv 6 \bmod 5$ & $r \equiv 4 \bmod 7$ & $(r-11)$ & 1 & 3 & 5 & 1 & 1
\end{tabular}

The Riemann-Hurwitz formula shows that $g$ is the genus of the Riemann surface $X=\mathcal{H} / \operatorname{ker} \theta$, since

$$
7=\frac{2 \operatorname{genus}(X)-2}{2+\left(\frac{g-8}{3}\right) \frac{6}{7}} .
$$

By construction the surface $X$ has an order 7 automorphism $\xi$ with $r=\frac{g-8}{3}$ fixed points. Using a similar argument to the one in the previous cases, the maximal number of fixed points of an automorphism of surfaces of genus 2 and the CastelnuovoSeveri inequality we have that $X$ has no automorphisms besides $C_{7}=\langle\xi\rangle$. The surfaces admitting a cyclic action as above form an isolated stratum of dimension $3 \times 2-3+r=\frac{g+1}{3}$.

Remark 3.2. There are several ways of defining the epimorphisms in the above theorem, and the isolated strata are not unique. For instance for $g \equiv 0 \bmod 3$ and $g \equiv 2 \bmod 7$, we can consider the following monodromy $\theta$ with generating vector $\left(n_{1} \alpha, n_{2} \alpha^{2}, n_{3} \alpha^{3}, n_{4} \alpha^{4}, n_{5} \alpha^{5}, n_{6} \alpha^{6}\right)$ where

\begin{tabular}{c|c|cccccc}
$g \bmod 7$ & $r \bmod 7$ & $n_{1}$ & $n_{2}$ & $n_{3}$ & $n_{4}$ & $n_{5}$ & $n_{6}$ \\
\hline$g \equiv 2 \bmod 7$ & $r \equiv 5 \bmod 7$ & $(r-3)$ & 1 & 0 & 1 & 0 & 1 \\
\hline
\end{tabular}

This epimorphism $\theta$ induces an action of a different topological type from the action considered in Case 1 of the proof of Theorem 3.1 (see [N] [H]). Hence $\theta$ determines an isolated stratum in $\mathcal{B}_{g}$ different from the one constructed in the proof of the Theorem 3.1.

In [BCI], using automorphisms of order 5 , we proved that the branch locus $\mathcal{B}_{g}$ of moduli spaces of Riemann surfaces of even genera at least 18 and genera congruent with $1 \bmod 4$ at least 29 are disconnected, with the exception of the genus 37 . We establish the results in [BCI] here for the sake of completedness.

Theorem 3.3. [BCI] $\mathcal{B}_{g}$ contains an isolated equisymmetric stratum of complex dimension:

1. $\frac{g}{2}-1$ if $g \equiv 0 \bmod 2$, for $g>16$,

2. $\frac{g-1}{2}$ if $g \equiv 1 \bmod 4$, for $g>25, g \neq 37$. 
Proof. We will construct monodromies $\theta: \Delta(h ; 5, . r ., 5) \rightarrow C_{5}$, where $r=\frac{g-5 h+4}{2}$, $h=0,1, \theta\left(x_{i}\right)=\alpha^{t_{i}}, i=1, \ldots, r$. Let $n_{j}=\left|\left\{t_{i}=j ; i=1, \ldots, r\right\}\right|$, then we will define the epimorphism $\theta$ by sending $n_{i}$ of the canonical elliptic generators of $\Delta$ to $\alpha^{i}$. If $h=1$ then $\theta\left(a_{1}\right)=\theta\left(b_{1}\right)=1$ since the class of the action does not depend on the images of the hyperbolic generators of $\Delta$. See $[N]$ and $[H]$.

Case 1. $g \equiv 0 \bmod 2$. Let $\Delta$ be a Fuchsian group with signature $(0 ; 5, . r ., 5)$, with $r=\frac{g+4}{2}$.

The monodromies will be given by the generating vector $\left(n_{1} \alpha, n_{2} \alpha^{2}, n_{3} \alpha^{3}, n_{4} \alpha^{4}\right)$. The values of $n_{j}$ for the different monodromies are given according to the congruence of $g$ modulus 5 in the following table:

\begin{tabular}{c|c|cccc}
$g \bmod 5$ & $r \bmod 5$ & $n_{1}$ & $n_{2}$ & $n_{3}$ & $n_{4}$ \\
\hline$g \equiv 0 \bmod 5$ & $r \equiv 2 \bmod 5$ & $(r-13)$ & 5 & 1 & 7 \\
\hline$g \equiv 1 \bmod 5$ & $r \equiv 0 \bmod 5$ & $(r-7)$ & 5 & 1 & 1 \\
\hline$g \equiv 2 \bmod 5$ & $r \equiv 3 \bmod 5$ & $(r-9)$ & 1 & 3 & 5 \\
\hline$g \equiv 3 \bmod 5$ & $r \equiv 1 \bmod 5$ & $(r-7)$ & 1 & 5 & 1 \\
\hline$g \equiv 4 \bmod 5$ & $r \equiv 4 \bmod 5$ & $(r-9)$ & 5 & 1 & 3
\end{tabular}

With one exception, in the case, $g=20, r=12$, we consider the epimorphism $\theta: \Delta(0 ; 5, .12,5) \rightarrow C_{5}$ defined by $\theta\left(x_{1}\right)=\alpha, \theta\left(x_{2}\right)=\cdots=\theta\left(x_{8}\right)=\alpha^{2}, \theta\left(x_{9}\right)=\alpha^{3}$, $\theta\left(x_{10}\right)=\cdots=\theta\left(x_{12}\right)=\alpha^{4}$.

Case 2. $g \equiv 1 \bmod 4, g \neq 37$. Let $\Delta$ be a Fuchsian group with signature $(0 ; 5, . r ., 5)$, with $r=\frac{g-1}{2}$.

The monodromies will be given by the generating vector $\left(1,1, n_{1} \alpha, n_{2} \alpha^{2}, n_{3} \alpha^{3}\right.$, $\left.n_{4} \alpha^{4}\right)$, where the 1 's in the generating vector mean that $\theta\left(a_{1}\right)=\theta\left(b_{1}\right)=1$. The values of $n_{j}$ for the different monodromies are given according to the congruence of $g$ modulus 5 in the following table:

\begin{tabular}{c|c|cccc}
$g \bmod 5$ & $r \bmod 5$ & $n_{1}$ & $n_{2}$ & $n_{3}$ & $n_{4}$ \\
\hline$g \equiv 0 \bmod 5$ & $r \equiv 2 \bmod 5$ & $(r-13)$ & 5 & 1 & 7 \\
\hline$g \equiv 1 \bmod 5$ & $r \equiv 0 \bmod 5$ & $(r-7)$ & 5 & 1 & 1 \\
\hline$g \equiv 2 \bmod 5$ & $r \equiv 3 \bmod 5$ & $(r-19)$ & 11 & 3 & 5 \\
\hline$g \equiv 3 \bmod 5$ & $r \equiv 1 \bmod 5$ & $(r-7)$ & 1 & 5 & 1 \\
\hline$g \equiv 4 \bmod 5$ & $r \equiv 4 \bmod 5$ & $(r-11)$ & 1 & 5 & 5
\end{tabular}

We see that the given epimorphisms satisfy the conditions that forced the automorphism of the quotient torus to be the identity except for $g=37, r=18$.

By construction the surfaces $X$ have an order 5 automorphism $\xi$ with $r=\frac{g-8}{3}$ fixed points. Using the same argument as in Theorem 3.1, the maximal number of fixed points of an automorphisms of surfaces of genus $h, h=0,1$ and the CastelnuovoSeveri inequality we have that, under this condition, $X$ has no automorphisms besides $C_{5}=\langle\xi\rangle$. The surfaces admitting a cyclic action as above form an isolated stratum of dimension $3 \times h-3+r=\frac{g+h-2}{2}$. Thus the dimension of the isolated strata is $\frac{g-2}{2}$ for even genera $(h=0)$, and $\frac{g-1}{2}$ for genera congruent with $1 \bmod 4(h=1)$.

As a consequence of Theorems 3.1 and 3.3 we have:

Corollary 3.4. $\mathcal{B}_{g}$ is disconnected for $g \geq 60$. 


\section{Genera with $\mathcal{B}_{g}$ connected}

We finally prove that the genera where $\mathcal{B}_{g}$ is connected are exactly $3,4,13,17,19$ and 59 . We begin proving that for these genera, in fact, $\mathcal{B}_{g}$ is connected.

Theorem 4.1. The branch locus $\mathcal{B}_{g}$ is connected for genera 3, 4, 7, 13, 17, 19 and 59 .

Proof. From Remark 2.1, it follows that we only need to look at actions of cyclic groups $C_{p}$, with $p$ a prime number, to determine that the branch locus is connected. We first show that each action of a cyclic group of prime order, given by the epimorphism $\theta: \Delta(h ; p, . \stackrel{r}{.}, p) \rightarrow C_{p}$, extends to an action of either $C_{2 p}$ or $D_{p}$. First, we know that strata corresponding to actions of order 2 or 3 belong to the same connected component [BI]. Thus we will only consider actions of prime order greater than 3. With the Riemann-Hurwitz formula it is easy to find the pairs of signatures and corresponding group orders.

The genera 3, 4 and 7 were studied in [CI2], [CI3], [BCIP] and [BI].

$\mathcal{B}_{13}$ : The automorphisms of prime order of Riemann surfaces of genus 13 have one of the following orders: 2, 3, 5, 7 or 13 .

$p=5$ : The only signature corresponding an actions of order 5 is $(1 ; 5, .6 ., 5)$. Let $\theta: \Delta(1 ; 5, .6 .6,5) \rightarrow C_{5}$ be defined by the generating vector $\left(1,1 ; \alpha^{t_{1}}, \ldots\right.$, $\left.\alpha^{t_{6}}\right)$. We may assume that $t_{1}=t_{2}$. Now let $\bar{\theta}: \bar{\Delta}(0 ; 5,10,10,10,10) \rightarrow C_{10}$ be defined by $\left(\bar{\alpha}^{2 t_{i}}, \bar{\alpha}^{t_{3}+5 \delta_{3}}, \ldots, \bar{\alpha}^{t_{6}+5 \delta_{6}}\right)$, where $\delta_{i} \not \equiv t_{i} \bmod 2$. We note that

$$
\sum t_{i} \equiv \mid\left\{t_{i} \mid t_{i} \text { is odd }\right\} \mid \bmod 2 \text { and } \mid\left\{t_{i} \mid t_{i} \text { is odd }\right\}|+|\left\{t_{i} \mid t_{i} \text { is even }\right\} \mid=6 \text {. }
$$

So it follows that

$$
\begin{aligned}
\sum t_{i}+5 \mid\left\{t_{i} \mid t_{i} \text { is even }\right\} \mid & \equiv \\
5 \mid\left\{t_{i} \mid t_{i} \text { is odd }|+5|\left\{t_{i} \mid t_{i} \text { is even }\right\} \mid\right. & \equiv 30 \equiv 0 \bmod 10 .
\end{aligned}
$$

Thus $\bar{\theta}$ is well-defined and $\left.\bar{\theta}\right|_{\Delta}=\theta$, see [Si1].

$p=7$ : The only signature is $(1 ; 7,7,7,7)$. Let $\theta: \Delta(1 ; 7,7,7,7) \rightarrow C_{7}$ be defined by the generating vector $\left(1,1 ; \alpha^{t_{1}}, \ldots, \alpha^{t_{4}}\right)$ and let $\bar{\theta}: \bar{\Delta}(0 ; 14,14,14,14)$ $\rightarrow C_{14}$ be defined by $\left(\bar{\alpha}^{t_{1}+7 \delta_{1}}, \ldots, \bar{\alpha}^{t_{4}+7 \delta_{4}}\right)$, where $\delta_{i} \not \equiv t_{i} \bmod 2$. Clearly, $\bar{\theta}$ is well-defined and $\left.\bar{\theta}\right|_{\Delta}=\theta$.

$p=13$ : The only signature is $(1 ; 13,13)$ and it is easy to see that any action $\theta$ is given by $\left(1,1 ; \alpha, \alpha^{-1}\right)$ and extends to an action of order 26 given by $\bar{\theta}: \Delta(0 ; 2,2,26,26) \rightarrow C_{26}$, defined by $\left(\alpha^{13}, \alpha^{13}, \alpha, \alpha^{-1}\right)$. See [Si2].

Since all the actions of order 5,7 and 13 can be extended to actions of $C_{10}, C_{14}$ and $C_{26}$ respectively, we have that $\overline{\mathcal{M}}_{13}^{C_{5}}, \overline{\mathcal{M}}_{13}^{C_{7}}$ and $\overline{\mathcal{M}}_{13}^{C_{13}}$ have non-empty intersection with $\overline{\mathcal{M}}_{13}^{C_{2}}$ and it follows that $\mathcal{B}_{13}$ is connected.

$\mathcal{B}_{17}$ : For Riemann surfaces of genus 17 , the automorphisms of prime order have one of the following orders; $2,3,5,7$ or 17 .

$p=5$ : The signatures are $(1 ; 5, .8 ., 5)$ and $(3 ; 5,5,5)$. Let $\theta: \Delta(1 ; 5, .8 ., 5) \rightarrow$ $C_{5}$ be defined by the generating vector $\left(1,1 ; \alpha^{t_{1}}, \ldots, \alpha^{t_{8}}\right)$. We may assume that $t_{1}=t_{2}$ and $t_{3}=t_{4}$. Now let $\bar{\theta}: \bar{\Delta}(0 ; 5,5,10,10,10,10) \rightarrow C_{10}$ be defined by $\left(\bar{\alpha}^{2 t_{1}}, \bar{\alpha}^{2 t_{3}}, \bar{\alpha}^{t_{5}+5 \delta_{5}}, \ldots, \bar{\alpha}^{t_{8}+5 \delta_{8}}\right)$, where $\delta_{i} \not \equiv t_{i} \bmod 2$. Clearly $2 t_{1}+2 t_{3}+$ 
$\sum t_{i}+5 \delta_{i} \equiv 0 \bmod 10$ and $\left.\bar{\theta}\right|_{\Delta}=\theta$. Now assume $\theta: \Delta(3 ; 5,5,5) \rightarrow C_{5}$ is defined by $\left(1, \ldots, 1 ; \alpha^{t_{1}}, \alpha^{t_{2}}, \alpha^{t_{3}}\right)$, then let $\bar{\theta}: \bar{\Delta}(0 ; 2, .5 ., 2,10,10,10) \rightarrow C_{10}$ be defined by $\left(\bar{\alpha}^{5}\right.$, dots, $\left.\bar{\alpha}^{5}, \bar{\alpha}^{t_{3}+5 \delta_{3}}, \bar{\alpha}^{t_{4}+5 \delta_{4}}, \bar{\alpha}^{t_{5}+5 \delta_{5}}\right)$ as before. It is clear that $\left.\bar{\theta}\right|_{\Delta}=\theta$.

$p=7$ : The only signature is $(2 ; 7,7,7)$. Every epimorphism from a group $\Delta(2 ; 7,7,7)$ onto $C_{7}$ extends to $\bar{\theta}: \Delta(0 ; 2,2,2,14,14,14) \rightarrow C_{14}$ similarly to the above cases.

$p=17$ : The only signature is $(1 ; 17,17)$ and any action extends to an action of order 34 with corresponding signature $(0 ; 2,2,34,34)$.

It follows that $\mathcal{B}_{17}$ is connected.

$\mathcal{B}_{19}$ : For Riemann surfaces of genus 19, the automorphisms of prime order have one of the following orders; 2, 3, 5, 7, 13 or 19.

$p=5$ : The signatures are $(1 ; 5, .9 ., 5)$ and $(3 ; 5,5,5,5)$. Let $\theta: \Delta(1 ; 5, .9 ., 5)$ $\rightarrow C_{5}$ be defined by the generating vector $\left(1,1 ; \alpha^{t_{1}}, \ldots, \alpha^{t_{9}}\right)$. We may assume that $t_{1}=t_{2}, t_{3}=t_{4}$ and $t_{5}=t_{6}$. Then let $\bar{\theta}: \bar{\Delta}(0 ; 2,5,5,5,10,10,10) \rightarrow C_{10}$ be defined by $\left(\bar{\alpha}^{5}, \bar{\alpha}^{2 t_{1}}, \bar{\alpha}^{2 t_{3}}, \bar{\alpha}^{2 t_{5}}, \bar{\alpha}^{t_{7}+5 \delta_{7}}, \ldots, \bar{\alpha}^{t_{9}+5 \delta_{9}}\right)$, where $\delta_{i} \not \equiv t_{i} \bmod 2$. Clearly $\sum s_{i} \equiv 0 \bmod 10$ and $\left.\bar{\theta}\right|_{\Delta}=\theta$. Now assume $\theta: \Delta(3 ; 5,5,5,5) \rightarrow C_{5}$ is defined by $\left(1, \ldots, 1 ; \alpha^{t_{1}}, \ldots, \alpha^{t_{4}}\right)$, then let $\bar{\theta}: \bar{\Delta}(0 ; 2, . .4 ., 2,10, .4 ., 10) \rightarrow C_{10}$ be defined by $\left(\bar{\alpha}^{5}, \ldots, \bar{\alpha}^{5}, \bar{\alpha}^{t_{1}+5 \delta_{1}}, \ldots, \bar{\alpha}^{t_{4}+5 \delta_{4}}\right)$ as before. It is clear that $\left.\bar{\theta}\right|_{\Delta}=\theta$.

$p=7$ : The only signature is $(1 ; 7, .6 ., 7)$. First, assume that an epimorphism $\theta: \Delta(1 ; 7, .6 ., 7) \rightarrow C_{7}$ is defined by the generating vector $\left(\alpha^{1}, \alpha^{2}, \alpha^{3}, \alpha^{4}, \alpha^{5}\right.$, $\left.\alpha^{6}\right)$. Then consider the epimorphism $\bar{\theta}: \Delta(0 ; 2, .4 ., 2,7,7,7) \rightarrow D_{7}$ defined by $\left(\sigma, \sigma, \sigma, \sigma \alpha, \alpha, \alpha^{2}, \alpha^{3}\right)$. We see that $\left.\bar{\theta}\right|_{\Delta}=\theta$. Note that in this case we must use a dihedral group to extend the action. Now let $\theta$ be defined by $\left(1,1 ; \alpha^{t_{1}}, \ldots, \alpha^{t_{6}}\right)$, assuming that $t_{1}=t_{2}$. Let $\bar{\theta}: \Delta(0 ; 7,14.4 ., 14) \rightarrow C_{14}$ be defined by $\bar{\theta}\left(y_{i}\right)=\bar{\alpha}^{s_{i}}$, where $s_{1}=2 t_{1}$ and $s_{i}=t_{i+1}+7 \delta_{i+1}, i=2, \ldots, 5$, where $\delta_{i} \not \equiv t_{i} \bmod 2$. It is clear that $\left.\bar{\theta}\right|_{\Delta}=\theta$.

$p=13$ : The only signature is $(1 ; 13,13,13)$. Every epimorphism $\theta: \Delta(1 ; 13$, $13,13) \rightarrow C_{13}$ extends to $\bar{\theta}: \Delta(0 ; 2,26,26,26) \rightarrow C_{26}$ similarly to above.

$p=19$ : The only signature is $(1 ; 19,19)$ and any action extends to an action of order 38 with signature $(0 ; 2,2,38,38)$.

It follows that $\mathcal{B}_{19}$ is connected.

$\mathcal{B}_{59}$ : For Riemann surfaces of genus 59, the automorphisms of prime order have one of the following orders; $2,3,5,7,11,17,29$ or 59.

$p=5$ : The signatures corresponding to actions of order 5 are $(1+2 j ; 5, \ldots-5,5)$, $j=0, \ldots, 5$. Let $\theta: \Delta(1+2 j ; 5,2, \cdots-5 j, 5) \rightarrow C_{5}$ be defined by the generating vector $\left(1, \ldots, 1 ; \alpha^{t_{1}}, \ldots, \alpha^{t_{29-5 j}}\right)$. We may assume that $t_{2 k-1}=t_{2 k}$, for $k=$ $1, \ldots,\left\lfloor\frac{26-5 j}{2}\right\rfloor$. Now let $\bar{\theta}: \bar{\Delta}(0 ; 2, \stackrel{1+j}{.}, 2,5, \stackrel{13-4 j}{*}, 5,10, \stackrel{3+3 j}{\cdot}, 10) \rightarrow C_{10}$ be defined by the generating vector $\left(\bar{\alpha}^{5}, \ldots, \bar{\alpha}^{5}, \bar{\alpha}^{s_{1}}, \ldots, \bar{\alpha}^{s_{13-4 j}}, \bar{\alpha}^{r_{1}}, \ldots, \bar{\alpha}^{r_{3+3 j}}\right)$, where $s_{i}=2 t_{2 i}$, for $i=1 \ldots 13-4 j$ and $r_{i}=t_{i+26-8 j}+5 \delta_{i}$, where $\delta_{i} \not \equiv t_{i} \bmod 2$. We note that

$\sum t_{i} \equiv \mid\left\{t_{i} \mid t_{i}\right.$ is odd $\} \mid \bmod 2$ and $\mid\left\{t_{i} \mid t_{i}\right.$ is odd $\}|+|\left\{t_{i} \mid t_{i}\right.$ is even $\} \mid=29-5 j$. 
So it follows that

$$
\begin{aligned}
5(1+j)+\sum s_{i}+\sum r_{i}=5(1+j)+\sum t_{i}+5 \mid\left\{t_{i} \mid t_{i} \text { is even }\right\} \mid & \equiv \\
5(1+j)+5 \mid\left\{t_{i} \mid t_{i} \text { is odd }\right\}|+5|\left\{t_{i} \mid t_{i} \text { is even }\right\} \mid & \equiv \\
5(1+j+29-5 j)=5(30-4 j) & \equiv 0 \bmod 10 .
\end{aligned}
$$

Thus $\bar{\theta}$ indeed defines an action of $C_{10}$ and $\theta$ extends to $\bar{\theta}$.

$p=7$ : The signatures corresponding to actions of order 7 are $\left(2+3 j ; 7_{, 17-7 j}^{17} 7\right)$, $j=0, \ldots, 2$. Let $\theta: \Delta(2+3 j ; 7, \ldots-7 j, 7) \rightarrow C_{7}$ be defined by the generating vector $\left(1, \ldots, 1 ; \alpha^{t_{1}}, \ldots, \alpha^{t_{17-7 j}}\right)$. First assume that $j=0$, we may then assume that $t_{2 k-1}=t_{2 k}$, for $k=1, \ldots, 6$. Let $\bar{\theta}: \bar{\Delta}\left(0 ; 2,7, .6 ., 7,14, .{ }^{5} ., 14\right) \rightarrow C_{14}$ be defined by the generating vector $\left(\bar{\alpha}^{7}, \bar{\alpha}^{s_{1}}, \ldots, \bar{\alpha}^{s_{13-4 j}}, \bar{\alpha}^{r_{1}}, \ldots, \bar{\alpha}^{r_{3+3 j}}\right)$, where $s_{i}=t_{2 i}, i=2 \ldots 6$, and $r_{i}=t_{i+12}+7 \delta_{i}$, where $\delta_{i} \not \equiv t_{i} \bmod 2$. We note that:

$\sum t_{i} \equiv \mid\left\{t_{i} \mid t_{i}\right.$ is odd $\} \mid \bmod 2$ and $\mid\left\{t_{i} \mid t_{i}\right.$ is odd $\}|+|\left\{t_{i} \mid t_{i}\right.$ is even $\} \mid=17$.

So it follows that

$$
\begin{aligned}
7+\sum s_{i}+\sum r_{i}=7+\sum t_{i}+7 \mid\left\{t_{i} \mid t_{i} \text { is even }\right\} \mid & \equiv \\
7+7 \mid\left\{t_{i} \mid t_{i} \text { is odd }\right\}|+7|\left\{t_{i} \mid t_{i} \text { is even }\right\} \mid & \equiv \\
7(1+17) & \equiv 0 \bmod 14 .
\end{aligned}
$$

For $j=1$ or 2 consider $\left.\bar{\theta}: \bar{\Delta}\left(0 ; 2,{ }_{13 j-11}, 2,14, \ldots, \ldots j\right) 14\right) \rightarrow C_{14}$ defined by the generating vector $\left(\bar{\alpha}^{7}, \ldots, \bar{\alpha}^{7}, \bar{\alpha}^{s_{1}}, \ldots, \bar{\alpha}^{s_{17-7 j}}\right)$, where $s_{i}=t_{i}+7 \delta_{i}$, such that $\delta_{i} \not \equiv t_{i} \bmod 2$. Now

$$
\begin{aligned}
7(13 j-11)+\sum s_{i}=7(13 j-11)+\sum t_{i}+7 \mid\left\{t_{i} \mid t_{i} \text { is even }\right\} \mid & \equiv \\
7(13 j-11)+7 \mid\left\{t_{i} \mid t_{i} \text { is odd }\right\}|+7|\left\{t_{i} \mid t_{i} \text { is even }\right\} \mid & \equiv \\
7(13 j-11+17-7 j)=7(6+6 j) & \equiv 0 \bmod 14 .
\end{aligned}
$$

Thus $\bar{\theta}$ indeed defines an action of $C_{14}$ in each case and $\theta$ extends to $\bar{\theta}$.

$p=11$ : The only actions of order 11 are $\theta: \Delta(4 ; 11, .5 ., 11) \rightarrow C_{11}$, defined by $\left(1, \ldots, 1 ; \alpha^{t_{1}}, \ldots, \alpha^{t_{5}}\right), t_{i} \in\{1, \ldots, 10\}$. Now, consider $\bar{\theta}: \bar{\Delta}(0 ; 2, . \stackrel{5}{.}, 2,22$, $.5,22) \rightarrow C_{22}$ be defined by $\left(1, \ldots, 1 ; \alpha^{11}, \ldots, \alpha^{11}, \alpha^{s_{1}}, \ldots, \alpha^{s_{5}}\right)$, where $s_{i}=t_{i}+11 \delta_{i}$, such that $\delta_{i} \not \equiv t_{i} \bmod 2$. As in the cases above we see that $\bar{\theta}$ indeed defines an action which extends the action induced by $\theta$.

$p=17$ : The only actions of order 17 are $\theta: \Delta(3 ; 17,17,17) \rightarrow C_{17}$, defined by $\left(1, \ldots, 1 ; \alpha^{t_{1}}, \alpha^{t_{2}}, \alpha^{t_{3}}\right)$. Let $\bar{\theta}: \bar{\Delta}(0 ; 2, .5 ., 2,34,34,34) \rightarrow C_{34}$ be defined by $\left(1, \ldots, 1 ; \alpha^{17}, \ldots, \alpha^{17}, \alpha^{s_{1}}, \ldots, \alpha^{s_{5}}\right)$, where $s_{i}=t_{i}+17 \delta_{i}$, such that $\delta_{i} \not \equiv t_{i} \bmod 2$. Again, we see that $\bar{\theta}$ indeed defines an action which extends $\theta$. $p=29$ : The only action of order 29 is $\theta: \Delta(3 ;-) \rightarrow C_{29}$ clearly extending to an action $\bar{\theta}: \bar{\Delta}(0 ; 2,2,2,2,2,2,2,2) \rightarrow D_{29}$.

$p=59$ : The only action of order 59 is $\theta: \Delta(1 ; 59,59) \rightarrow C_{59}$ clearly extending to an action $\bar{\theta}: \bar{\Delta}(0 ; 2,2,2,59) \rightarrow D_{59}$.

So finally, $\mathcal{B}_{59}$ is connected.

Theorem 4.2. The branch locus $\mathcal{B}_{g}$ is disconnected for every genus $g$ different from $3,4,7,13,17,19$, and 59 . 
In fact, the branch locus $\mathcal{B}_{g}$ is connected if and only if $g \in\{3,4,7,13,17,19,59\}$.

Proof. The following cases have been previously studied:

(i) For genus 3, a proof of the connectedness of the branch locus is given in [BCIP].

(ii) For genera 5 to 9 , a proof is in $[\mathrm{BI}]$, for even genera $g \geq 18$ and genera $g \equiv 1$ $\bmod 4, g \geq 29$ a proof is in [BCI].

(iii) For genera a multiple of three at least 39, genera $g \equiv 1 \bmod 3, g \geq 50$ and genera $g \equiv 2 \bmod 3, g \geq 65$ is proved in Theorems 3.1 and 3.3.

(iv) $\mathcal{B}_{g}, g=11,14,15,21,23,35$ contain isolated points since $2 g+1$ is a prime number. See Kulkarni [K].

(v) $\mathcal{B}_{g}, g=10,12,16$ contain isolated strata of dimension 1 since $g+1$ is a prime number. See [CI4].

Now, we prove that $\mathcal{B}_{g}$ is disconnected for the remaining genera: 25, 27, 31, 37 and 43. For these genera we will find isolated strata by defining monodromies $\theta: \Delta \rightarrow C_{p}$ of maximal actions of cyclic groups of prime order. We will construct epimorphisms $\theta: \Delta(h ; p, . r ., p) \rightarrow C_{p}$ that do not extend. To show this we consider potential extensions $\bar{\theta}: \bar{\Delta} \rightarrow G$ where $C_{p}$ is a maximal subgroup of some group $G$ (since any other extension will have such a group as a subgroup). This is done here with the computational algebra system GAP [GAP]. See the Appendix A for examples of used GAP codes.

$\mathcal{B}_{25}$ : Consider the epimorphism $\theta: \Delta(0 ; 11, .7 ., 11) \rightarrow C_{11}=\langle\alpha\rangle$ with generating vector $\left(\alpha, \alpha^{2}, \alpha^{4}, \alpha^{5}, \alpha^{6}, \alpha^{7}, \alpha^{8}\right)$. By Singerman's results ([Si1]) we can calculate all signatures of possible extensions of this action. Now the only possible extensions $\bar{\theta}: \bar{\Delta} \rightarrow G$ that we need to check are the ones such that $C_{11}$ is a maximal subgroup of $G$. With the use of GAP we can easily find that the groups are $C_{22}, C_{33}, C_{55}$ and $C_{77}$, with signatures $(0 ; 2,11,11,11,22)$, $(0 ; 3,11,11,33),(0 ; 11,55,55)$ and $(0 ; 7,7,11)$ respectively. The image of each generator in $\bar{\Delta}$ of order 11 will induce two, three, five or seven generators of $\Delta$ with the same image $\left(\alpha^{i}\right)$, this fact contradicts that $\bar{\theta}$ is an extension of $\theta$.

$\mathcal{B}_{27}$ : Consider the epimorphism $\theta: \Delta(0 ; 19, .5 ., 19) \rightarrow C_{19}=\langle\alpha\rangle$ with generating vector $\left(\alpha \alpha^{3}, \alpha^{4}, \alpha^{5}, \alpha^{6}\right)$. By results in [Si1] we can calculate all signatures of possible extensions, $\bar{\theta}: \bar{\Delta} \rightarrow G$, of this action, which are the following:

$(0 ; 2,19,19,38)$, of index 2 ,

$(0 ; 19,57,57)$, of index 3 ,

$(0 ; 4,19,76)$, of index 4 ,

$(0 ; 5,5,19)$, of index 5 ,

$(0 ; 2,38,57)$, of index 6 ,

$(0 ; 2,5,38)$, of index 10 , and

$(0 ; 2,3,19)$, of index 24 .

However, groups of the given orders with maximal subgroups corresponding to $C_{19}$, such that there exists generating vectors with the given signatures, are $C_{38}, C_{57}$ and $C_{95}$. Note that each generator of $\bar{\Delta}$ of order 19 will induce two, three or five generators of $\Delta$ with the same image $\left(\alpha^{i}\right)$, this fact contradicts that $\bar{\theta}$ is an extension of $\theta$.

$\mathcal{B}_{31}$ : Consider the epimorphism $\theta: \Delta(1 ; 13, . .5 ., 13) \rightarrow C_{13}=\langle\alpha\rangle$ with generating vector $\left(\alpha^{-1}, \alpha^{2}, \alpha^{3}, \alpha^{4}, \alpha^{5}\right)$. By [Si1] we can calculate all signatures of possible 
extensions of this action. Now the only possible extensions $\bar{\theta}: \bar{\Delta} \rightarrow G$ that we need to check are the ones such that $C_{13}$ is a maximal subgroup of $G$. With the use of GAP we can easily find that the groups are $C_{26}$ and $C_{39}$, with signatures $(0 ; 2,13,26,26,26),(0 ; 2,2,2,13,13,26)$ and $(0 ; 3,13,39,39)$ respectively. The image of each generator in $\bar{\Delta}$ of order 13 will induce two or three generators of $\Delta$ with the same image $\left(\alpha^{i}\right)$, this fact contradicts that $\bar{\theta}$ is an extension of $\theta$.

$\mathcal{B}_{37}$ : Consider the epimorphism $\theta: \Delta(1 ; 13, .6 ., 13) \rightarrow C_{13}=\langle\alpha\rangle$ with generating vector $\left(\alpha^{-1}, \alpha^{2}, \alpha^{3}, \alpha^{4}, \alpha^{5}\right)$. By [Si1] we can calculate all signatures of possible extensions of this action. Now, the only possible extensions $\bar{\theta}: \bar{\Delta} \rightarrow G$ we need to check are the ones such that $C_{13}$ is a maximal subgroup of $G$. With the use of GAP we can easily find that the groups and signatures are:

$C_{26}$ with signatures $(0 ; 13,26,26,26,26),(0 ; 2,2,13,13,26,26),(0 ; 2,2,2,2$, $13,13,13)$ and $(1 ; 13,13,13)$.

$D_{13}$ with signatures $(0 ; 2,2,2,2,13,13,13)$ and $(1 ; 13,13,13)$.

$C_{39}$ with signatures $(0 ; 13,39,39,39),(0 ; 3,3,3,13,13)$ and $(1 ; 13,13)$ and

$C_{13} \rtimes C_{3}$, with signatures $(0 ; 3,3,3,13,13)$ and $(1 ; 13,13)$.

In the cyclic cases the image of each generator in $\bar{\Delta}$ of order 13 will induce two or three generators of $\Delta$ with the same image $\left(\alpha^{i}\right)$, which clearly is a contradiction. In the dihedral case the image of each generator in $\bar{\Delta}$ of order 13 will induce an element and its inverse $\left(\alpha^{i}\right.$ and $\left.\alpha^{-i}\right)$ and for $C_{13} \rtimes C_{3}$ the image of each generator in $\bar{\Delta}$ of order 13 will induce a triple $\left(\alpha^{i}, \alpha^{3 i}, \alpha^{9 i}\right)$, contradicting being extensions.

$\mathcal{B}_{43}$ : Consider the monodromy $\Delta(1 ; 13, .7 ., 13) \rightarrow C_{13}$ and the generating vector $\left(\alpha, \alpha^{2}, \alpha^{3}, \alpha^{4}, 2 \alpha^{5}, \alpha^{6}\right)$. Possible extensions $\bar{\theta}: \bar{\Delta} \rightarrow G$ with $C_{13}$ as a maximal subgroup of $G$ are given by the groups $C_{26}$ and $C_{39}$ with corresponding signatures $(0 ; 2 ; 13,13,26,26,26),(0 ; 2,2,2,13,13,13,26)$ and $(0 ; 3,3,13,13,39)$ respectively. Note that each generator in $\bar{\Delta}$ of order 13 will induce two or three elements with the same image $\left(\alpha^{i}\right)$, contradicting the fact of being extensions.

Question. In [BI] it is proved that all the strata $\mathcal{M}_{g}^{C_{2}}$ and $\mathcal{M}_{g}^{C_{3}}$, induced by actions of order 2 and 3, belongs to the same connected component of $\mathcal{B}_{g}$. Bartolini [Ba] showed that, up to genus 24 , the only connected components of $\mathcal{B}_{g}$ not containing $\mathcal{M}_{g}^{C_{2}}$ are formed by one equisymmetric stratum. We have found here connected components of $\mathcal{B}_{g}$ consisting of just one equisymmetric stratum. The question one rises is:

Is there a connected component of $\mathcal{B}_{g}$, other than the one containing $\mathcal{M}_{g}^{C_{2}}$ and $\mathcal{M}_{g}^{C_{3}}$, that contains more than just one equisymmetric stratum?

\section{Appendix A. GAP code}

We give a collection of GAP code examples, and the output when running an example, used for the case of $\mathcal{B}_{25}$. The other cases in Theorem 4.2 use similar codes. First we find the possible signatures corresponding to an extension of an action of order 11. Then we check the possible groups of the given orders, such that $C_{11}$ is a maximal subgroup. 


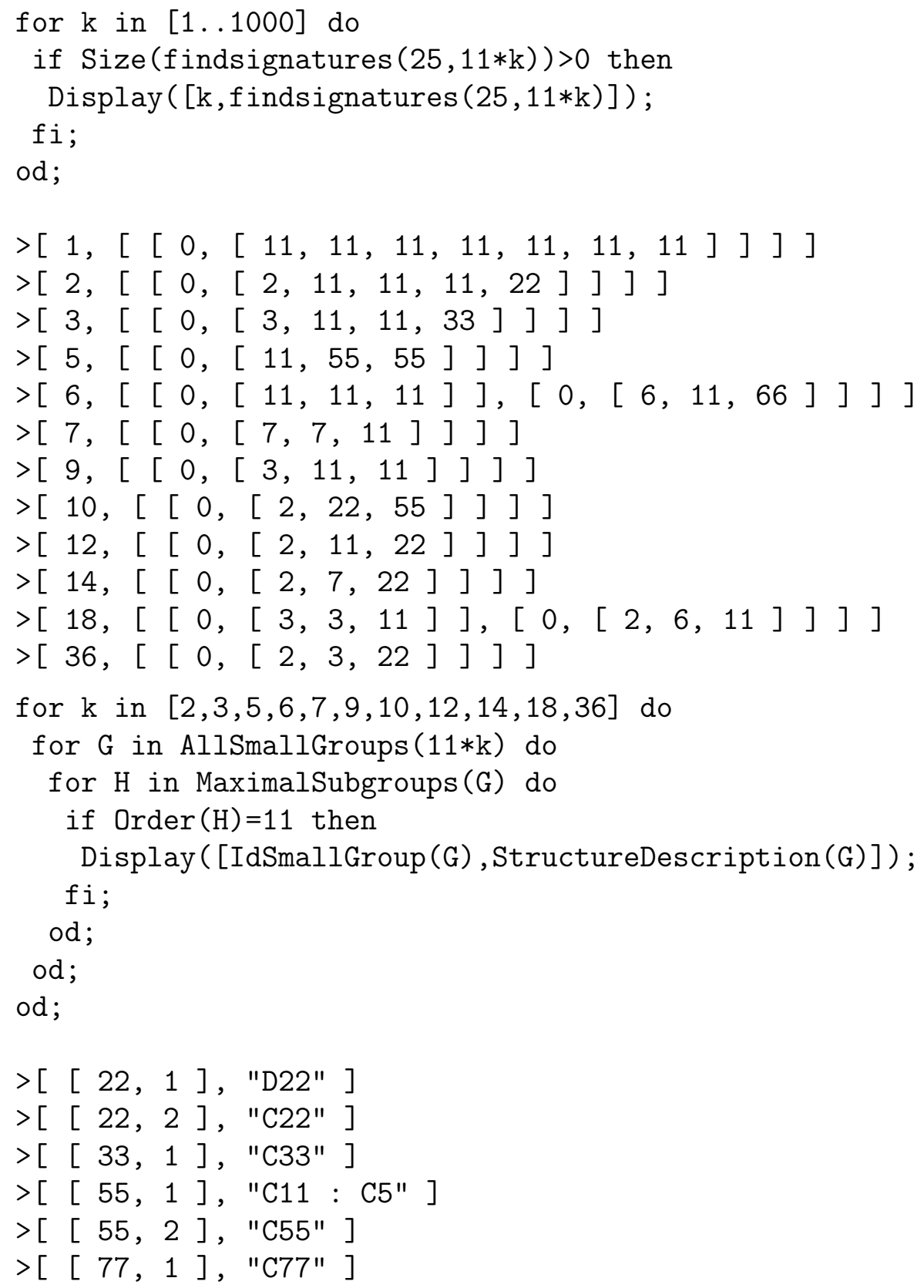

\section{References}

[A] Accola, R. D. M.: Topics in the theory of Riemann surfaces. - Lecture Notes in Math. 1595, Springer-Verlag, Berlin-Heidelberg, 1994.

[Ba] Bartolini, G.: On branch loci of moduli space of Riemann surfaces. - Ph.D. Thesis, Linköping Studies in Science and Technology, Dissertations 1440, 2012.

[BCI] Bartolini, G., A.F. Costa, and M. Izquierdo: On the connectivity of the branch locus of the moduli space of pentagonal Riemann surfaces. - Contemp. Math. 572, 2012, $19-24$.

[BCIP] Bartolini, G., A. F. Costa, M. Izquierdo, and A. M. Porto: On the connectedness of the branch locus of the moduli space of Riemann surfaces. - Rev. R. Acad. Cienc. Exactas Fís. Nat. Ser. A Mat. RACSAM 104:1, 2010, 81-86. 
[BI] Bartolini, G., and M. IzQuierdo: On the connectedness of branch loci of moduli spaces of Riemann surfaces of low genus. - Proc. Amer. Math. Soc. 140, 2012, 35-45.

[Bo] BolzA, O.: On binary sextics with linear transformations between themselves. - Amer. J. Math. 10, 1888, 47-70.

[B] Broughton, S. A.: The equisymmetric stratification of the moduli space and the Krull dimension of mapping class groups. - Topology Appl. 37, 1990, 101-113.

[BuCI $]$ Bujalance, E., A.F. Costa, and M. Izquierdo: A note on isolated points in the branch locus of the moduli space of compact Riemann surfaces. - Ann. Acad. Sci. Fenn. Math. 23:1, 1998, 25-32.

[BemS] Bujalance, E., J. J. Etayo, E. Martinez, and B. Szepietowski: The full group of automorphisms of non-orientable, unbordered Klein surfaces of topological genus 3, 4 and 5. - Preprint, 2011.

[BSS] Buser, P., M. SeppäLÄ, and R. Silhol: Triangulations and moduli spaces of Riemann surfaces with group actions. - Manuscripta Math. 88, 1995, 209-224.

[C] Cornalba, M.: On the locus of curves with automorphisms. - Ann. Mat. Pura Appl. (4) 149, 1987, 135-151; Erratum. - Ann. Mat. Pura Appl. (4) 187, 2008, 185-186.

[CI1] Costa, A. F., and M. Izquierdo: On the connectedness of the locus of real Riemann surfaces. - Ann. Acad. Sci. Fenn. Math. 27, 2002, 341-356.

[CI2] Costa, A. F., and M. Izquierdo: On the connectedness of the branch locus of the moduli space of Riemann surfaces of genus 4. - Glasg. Math. J. 52:2, 2010, 401-408.

[CI3] Costa, A.F., and M. Izquierdo: Equisymmetric strata of the singular locus of the moduli space of Riemann surfaces of genus 4. - LMS Lect. Note Series 368, 2010, 130-148.

[CI4] Costa, A. F., and M. Izquierdo: On the existence of connected components of dimension one in the branch loci of moduli spaces of Riemann surfaces. - Math. Scand. 111, 2012, $1-12$.

[GAP] The GAP-Group: GAP - Groups, algorithms, and programming, Version 4.4.12. - http:// www.gap-system.org, 2008.

[G] GonzÁlez-Díez, G.: On prime Galois covering of the Riemann sphere. - Ann. Mat. Pura Appl. 168, 1995, 1-15.

[H] Harvey, W.: On branch loci in Teichmüller space. - Trans. Amer. Math. Soc. 153, 1971, 387-399.

[K] KUlKarni, R. S.: Isolated points in the branch locus of the moduli space of compact Riemann surfaces. - Ann. Acad. Sci. Fenn. Ser. A I Math. 16, 1991, 71-81.

[MS] Macbeath, A. M., and D. Singerman: Spaces of subgroups and Teichmüller space. Proc. London Math. Soc. 31, 1975, 211-256.

[N] Nielsen, J.: Die Struktur periodischer Transformationen von Flächen. - Danske Vid Selsk. Mat-Fys. Medd. 1, 1937, 1-77.

[R] RAuch, H. E.: The singularities of the modulus space. - Bull. Amer. Math. Soc. 68, 1962, 390-394.

[Se] SEPpÄLÄ, M.: Real algebraic curves in the moduli space of complex curves. - Comp. Math. 74, 1990, 259-283.

[Si1] Singerman, D.: Subgroups of Fuchsian groups and finite permutation groups. - Bull. London Math. Soc. 2, 1970, 319-323.

[Si2] Singerman, D.: Finitely maximal Fuchsian groups. - J. London Math. Soc. (2) 6, 1972, $29-38$.

Received 22 March 2012 • Accepted 8 October 2012 